\title{
Systematic Review \\ Molecular Targets for Biological Therapies of Severe Asthma: Focus on Benralizumab and Tezepelumab
}

\author{
Shih-Lung Cheng ${ }^{1,2}$ \\ 1 Department of Internal Medicine, Far Eastern Memorial Hospital, Taipei 10042, Taiwan; \\ shihlungcheng@gmail.com; Tel.: +886-2-89667000; Fax: +886-2-77380708 \\ 2 Department of Chemical Engineering and Materials Science, Yuan Ze University, \\ Taoyuan City 320315, Taiwan
}

Citation: Cheng, S.-L. Molecular Targets for Biological Therapies of Severe Asthma: Focus on Benralizumab and Tezepelumab. Life 2021, 11, 744. https://doi.org/ $10.3390 /$ life11080744

Academic Editors: Laura Bergantini and Miriana d'Alessandro

Received: 30 June 2021

Accepted: 19 July 2021

Published: 26 July 2021

Publisher's Note: MDPI stays neutral with regard to jurisdictional claims in published maps and institutional affiliations.

Copyright: (C) 2021 by the author. Licensee MDPI, Basel, Switzerland. This article is an open access article distributed under the terms and conditions of the Creative Commons Attribution (CC BY) license (https:// creativecommons.org/licenses/by/ $4.0 /)$.

\begin{abstract}
Asthma is a heterogeneous respiratory disease characterized by usually reversible bronchial obstruction, which is clinically expressed by different phenotypes driven by complex pathobiological mechanisms (endotypes). In recent years several molecular effectors and signaling pathways have emerged as suitable targets for biological therapies of severe asthma, refractory to standard treatments. Indeed, various therapeutic mono-clonal antibodies currently allow one to intercept at different levels the chain of pathogenic events leading to type 2 (T2) airway inflammation. Pro-allergic immunoglobulin $\mathrm{E}$ (IgE) is the first molecule against which an anti-asthma monoclonal antibody (omalizumab) was developed; today other targets are successfully being exploited by biological treatments for severe asthma. In particular, pro-eosinophilic interleukin 5 (IL-5) can be targeted by mepolizumab or reslizumab, whereas benralizumab is a selective blocker of IL-5 receptor, and IL-4 and IL-13 can be targeted by dupilumab. Besides these drugs, which are already available in medical practice, other biologics are under clinical development such as those targeting innate cytokines, including the alarmin thymic stromal lymphopoietin (TSLP), which plays a key role in the pathogenesis of type 2 asthma. Therefore, ongoing and future biological therapies are significantly changing severe asthma management on a global level. These new therapeutic options make it possible to implement phenotype/endotype-specific treatments, which are delineating personalized approaches precisely addressing the individual traits of asthma pathobiology. The aim of the study is to review the immunopathology and treatment efficacy for severe asthma and focused on new biological agents with benralizumab (anti-IL-5) and tezepelumab (anti-TSLP).
\end{abstract}

Keywords: severe asthma; interleukin-5; thymic stromal lymphopoietin

\section{Introduction}

Based on GINA guidelines, severe asthma is a subset of difficult-to-treat asthma [1] Difficult-to-treat asthma is uncontrolled asthma despite the following of GINA Step 4 or 5 treatment. Uncontrolled asthma includes one or both of the following: (a) poor symptom control, (b) at least 2 exacerbations requiring oral corticosteroids (OCS) annually, or at least 1 serious exacerbation requiring hospitalization annually.

Current biologics are mainly targeting T2-high severe asthma, which is characterized by increased level of type 2 inflammation in the airway [2]. It manifests clinically with a combination of peripheral eosinophilia, sputum eosinophilia, and/or elevated fractional exhaled nitric oxide (FeNO) [3].

These biologics target interleukin-5 (IL-5) or interleukin-5 receptor (IL-5R), and thymic stromal lymphopoietin (TSLP). TSLP which is the upstream role in the asthma cascade, inhibiting its stimulating activity on dendric cells and innate lymphoid cells thus preventing the induction of type 2 cytokines (e.g., IL-5, IL-4, and IL-13) [4].

Benralizumab is a humanized, afucosylated, IgG1k isotype monoclonal antibody which specifically binds to interleukin-5 receptor alpha-directed cytolytic (IL-5R $\alpha$ ), which is 
expressed on eosinophils and basophils [5]. Benralizumab is uniquely engineered to recruit natural killer cells directly to its target, resulting in apoptosis via antibody-dependent cellular cytotoxicity, producing rapid and sustained complete depletion of eosinophils in blood and target tissues [6]. Its efficacy and safety have been confirmed in pivotal randomized clinical trials and long-term extension study (SIRROCO [7], CALIMA [8], BORA [9], and ZONDA [10]). Benralizumab $30 \mathrm{mg}$ every 8 weeks (Q8W); first three doses every 4 weeks $(\mathrm{Q} 4 \mathrm{~W})$ is indicated for the add-on-maintained treatment of patients with severe asthma aged 12 years and older, and with an eosinophilic phenotype. Benralizumab treatment enabled patients with severe, uncontrolled OCS-dependent asthma and baseline blood eosinophil counts $\geq 150$ cells $/$ uL to achieve and maintain asthma control while reducing OCS dosages [10]. The ANDHI study increases confidence in the benralizumab mechanism of action for treating patients with severe eosinophilic asthma through further assessment of the onset and maintenance of clinical effects, benefits in health-related quality of life (HRQOL) measures, and the potential to treat symptoms of nasal polyposis for patients with chronic rhinosinusitis with nasal polyposis [11]. Treatment with benralizumab for patients with severe eosinophilic asthma (BEC $\geq 150$ cells per $\mu \mathrm{L}$ ) significantly reduced the risk of asthma exacerbation, which was primarily driven by patients with efficacy associated with known markers of the eosinophilic phenotype.

Tezepelumab binds to TSLP, which is one of the key drivers of the asthmatic pathophysiology as it is produced by the airway epithelium in response to inhaled allergens and proinflammatory stressors [12]. Because of its upstream activity early in the inflammatory cascade, tezepelumab may have a role in patients with severe, uncontrolled asthma irrespective of patient phenotype or T2 biomarker status. In the phase $2 \mathrm{~b}$ PATHWAY trial the annualized rate of asthma exacerbations was up to $71 \%$ lower with tezepelumab than with placebo among patients with severe, uncontrolled asthma [12]. Furthermore, exacerbations were reduced regardless of baseline levels of inflammatory biomarkers, including fraction of exhaled nitric oxide (FeNO), blood eosinophils, IgE, and allergic status [12-14].

The UK Severe Asthma Registry (UKSAR) study demonstrated even though 68.9\% were prescribed biologic therapies including mepolizumab (50.3\%), benralizumab (26.1\%) and omalizumab (22.6\%), 51.7\% of the UKSAR remain poorly controlled. They continue to have a high exacerbation rate averaging four acute OCS courses/year, with an average ACQ6 of 2.9 at assessment, and on maintenance OCS. Treatment goals in asthma include symptom control and reducing risk of future exacerbations. However, approximately $3 \%$ to $5 \%$ of asthmatic patients have severe asthma where either symptoms persist or numerous exacerbation occur despite maximal treatment, an estimate that varies by country and may reach $\geq 10 \%$ in the United States [15].

Systemic reviews have been carried out for severe asthma in omalizumab, mepolizumab, reslizumab, benralizumab, and dupilumab from 2017 to 2021 [3,16-19]; however, only one of them has been reviewed in both benralizumab and tezepelumab [19]. The study was conducted in 2020, which did not include phase 3 NAVIGATOR study for tezepelumab [20], or phase 3b ANDHI study for benralizumab [11]. An Italian cross-sectional study analyzed real-life descriptions of severe refractory population from June 2017 to June 2019 [21]. Between patients in therapy with omalizuamb, six switched to bronchial thermoplasty, two shifted to mepolizumab, and two to benralizumab [21]. An Australia case report documenting a 68-year-old man revealed refractory airway eosinophilia after treatment with mepolizumab, but he then responded to benralizumab [22]. Another Italian real world study was carried out from January 2019 to November 2019. Forty-two benralizumab patients showed improved asthma control and lung function and a reduced OCS use among those previously treated with either omalizumab $(n=15)$ or mepolizumab $(n=5)$ or both omalizumab and mepolizumab $(n=2)$ [23]. According to a real-world study, physicians may prescribe benralizumab while omalizumab or mepolizumab are not adequately respond in clinical practice. Therefore, this systemic review is focused on benralizumab and tezepelumab. 
The objective of this study was to survey and elucidate the efficacy of benralizumab and tezepelumab using literature reviews on the assessment of symptom control, emergency department visits (severe acute exacerbations), lung function, and safety in those with severe uncontrolled asthma.

\section{Materials and Methods:}

The study followed the Preferred Reporting Items for Systemic Reviews and MetaAnalysis (PRISMA) guidelines.

\subsection{Search Strategy}

The systemic review is performed through independent searches of the MEDLINE, and the Cochrane Library database using free text search terms from inception to April 2021 and evaluated the title and abstract for eligibility. By performing a systemic literature review, 32 studies were identified. Among these, 30 studies were identified based on patients, interventions comparisons, outcomes, and study design (PICOs) criteria.

(A) Population: severe asthma.

(B) Intervention: tezepelumab or benralizumab for treatment of severe asthma

(C) Comparisons: not specific.

(D) Outcomes: symptom control, emergency department visits, lung function, and safety.

(E) Study design: clinical study, clinical trial, clinical trial, phase I, clinical trial, phase

II, clinical trial, phase III, clinical trial, phase IV, controlled clinical trial, multicenter study, observational study, pragmatic clinical trial, and randomized controlled trial.

\subsection{Study Selection}

Studies that met following criteria were excluded:

1. Review articles, case reports, and conference abstracts; and

2. Articles where the full texts were unavailable.

\subsection{Data Extraction}

The reviewer read the full text, supplementary, and appendix and extracted the data independently and meticulously. The following descriptive data were obtained from all included studies: first author, publication year, study phase, study locations, patient characteristics, methods, duration, and intervention. The reviewer checked the accuracy of data extraction.

\subsection{Summary Measures and Synthesis Results}

Main results are described narratively and tabulated as a summary of findings. Binary outcomes were presented at risk ratio (RR) and confidence interval, whereas continuous outcomes were presented at mean difference (MD) and 95\% CI. For each outcome, the change from baseline to the end of treatment vs. placebo were assessed (Tables S6-S11).

\section{Results and Discussions}

\subsection{Study Selection}

A total of 382 publications were identified from PubMed, while using the filters stated in study design, 32 studies remained. The search strategy is in Table S2, with search date on 10 April 2021. Of these, three were excluded for study populations. One study was added as it was published on May 13. There are 12 clinical trials and four observational studies (Table S3), 14 post-analysis (Table S4). The total 30 studies are listed in Table S5.

\section{Baseline Demographics and Clinical Characteristics}

Baseline demographics are presented in Table S5. Patient characteristics, such as age, race, gender, and BMI were included. Clinical characteristics, such as forced expiratory volume in $1 \mathrm{~s}$ (FEV1) on dosing date, Asthma control questionnaire 6 (ACQ-6), Asthma Quality of Life questionnaire for persons 12 years of age or older (AQLQ+12 score) were included. 


\subsection{Severe Exacerbations}

\subsubsection{Benralizumab}

The Cochrane review which included Bleeker 2016, Castro 2014, and Fitzgerald 2016 demonstrated benralizumab decreased annual exacerbation rates by $38 \%$ (rate ratio $=0.62$, $95 \%$ confidence interval 0.55 to 0.70$)$ vs. placebo $\left(n=2456, \mathrm{I}^{2}=0.0 \%\right)$ [16]. Based on the meta-analysis, eosinophilic group decreased exacerbations by $41 \%$ (rate ratio $=0.59,95 \% \mathrm{CI}$ 0.51 to 0.68 , which is larger than non-eosinophilic group (rate ratio $0.69,95 \% \mathrm{CI} 0.56$ to 0.85 ) without statistical difference $\left(p=0.22, \mathrm{I}^{2}=33.9 \%\right)$. On the other hand, for patients with blood eosinophil counts $<150$ cells $/ \mu \mathrm{L}$, Goldman et al. showed exacerbations reduction is not statistically significant ( $p=0.287$ in SIROCCO, $p=0.105$ in CALIMA) [24]. Other than eosinophilic subgroups, Ohta demonstrated benralizumab decreased exacerbations by $83 \%$ (Q8W, rate ratio $=0.17,95 \%$ CI 0.05 to 0.60 ) in Japanese patients [25]. In eosinophilic asthma ( $\geq 300$ cells/lL) patients, Chipps et al. [26] and Jackson et al. [27] demonstrated subgroups in atopic status and IgE. For patients who met the atopy and IgE criteria, benralizumab Q8W decreased exacerbation by $46 \%$ vs. placebo [26]. Jackson et al. showed across baseline serum IgE concentration quartiles, benralizumab Q8W resulted in $44 \%$ to $53 \%$ decreases in exacerbation rates $(p \leq 0.0057)$ [27]. Chipps indicated benralizumab decreased exacerbations significantly regardless of fixed airflow obstructions (FAO) status [28]. DuBuske et al. demonstrated rate reductions in seasonal marginal annual exacerbation rates were 37 to $50 \%$ versus placebo at each season $(p<0.001)$ [29]. (Table 1$)$.

Table 1. Summary of annual exacerbation rate-banralizumab.

\begin{tabular}{|c|c|c|c|c|c|c|}
\hline Trial & \multicolumn{3}{|c|}{ Treatment Arm } & Subjects & $\begin{array}{c}\text { Relative Reduction } \\
\text { vs. Placebo }\end{array}$ & $p$ Value vs. \\
\hline \multirow{3}{*}{ Bleecker et al., 2016} & \multirow{8}{*}{\multicolumn{2}{|c|}{ Eosinophilic }} & Placebo & 267 & - & - \\
\hline & & & Benralizumab 30 mg Q4W & 275 & $0.55(0.42 \text { to } 0.71)^{1}$ & $<0.0001$ \\
\hline & & & Benralizumab 30 mg Q8W & 267 & $0.49(0.37 \text { to } 0.64)^{1}$ & $<0.0001$ \\
\hline \multirow{3}{*}{ FitzGerald et al., 2016} & & & Placebo & 248 & - & - \\
\hline & & & Benralizumab 30 mg Q4W & 241 & 0.64 (0.49 to 0.85$)$ & 0.0018 \\
\hline & & & Benralizumab 30 mg Q8W & 239 & $0.72(0.54$ to 0.95$)$ & 0.0188 \\
\hline \multirow{2}{*}{ Castro et al., 2014} & & & Placebo & 83 & & \\
\hline & & & Benralizumab $20 \mathrm{mg}$ & 70 & $0.57(0.42-0.77)^{2}$ & 0.015 \\
\hline Subtotal $(95 \% \mathrm{CI})$ & & & & & $0.59(0.51-0.68)$ & NR \\
\hline \multirow{3}{*}{ Bleecker et al., 2016} & \multirow{6}{*}{\multicolumn{2}{|c|}{ Non-Eosinophilic }} & Placebo & 140 & - & - \\
\hline & & & Benralizumab 30 mg Q4W & 124 & $0.70(0.50 \text { to } 1.00)^{1}$ & 0.0471 \\
\hline & & & Benralizumab $30 \mathrm{mg}$ Q8W & 131 & $0.83(0.59 \text { to } 1.16)^{1}$ & 0.2685 \\
\hline \multirow{3}{*}{ FitzGerald et al., 2016} & & & Placebo & 122 & - & - \\
\hline & & & Benralizumab 30 mg Q4W & 116 & 0.64 (0.45 to 0.92$)$ & 0.015 \\
\hline & & & Benralizumab 30 mg Q8W & 125 & $0.60(0.42$ to 0.86$)$ & 0.0048 \\
\hline Subtotal $(95 \%$ CI $)$ & & & & & $0.69(0.56-0.85)$ & NR \\
\hline Total $(95 \% \mathrm{CI})$ & & & & & $0.62(0.55-0.70)$ & NR \\
\hline \multirow{8}{*}{ Goldman et al., 2017} & \multirow{4}{*}{$\begin{array}{l}\text { blood } \\
\text { eosinophils } \\
\geq 150 \text { cells } \\
\text { per uL }\end{array}$} & \multirow{2}{*}{ [SIROCCO] } & Placebo & 306 & - & - \\
\hline & & & Benralizumab 30 mg Q8W & 325 & $0.58(0.46$ to 0.74$)$ & $<0.001$ \\
\hline & & \multirow{2}{*}{ [CALIMA] } & Placebo & 315 & - & - \\
\hline & & & Benralizumab 30 mg Q8W & 300 & $0.64(0.50$ to 0.81$)$ & $<0.001$ \\
\hline & \multirow{4}{*}{$\begin{array}{c}\text { blood } \\
\text { eosinophils }< \\
150 \text { cells per uL }\end{array}$} & \multirow{2}{*}{ [SIROCCO] } & Placebo & 74 & - & - \\
\hline & & & Benralizumab 30 mg Q8W & 48 & $0.76(0.45$ to 1.27$)$ & 0.287 \\
\hline & & \multirow{2}{*}{ [CALIMA] } & Placebo & 40 & - & - \\
\hline & & & Benralizumab 30 mg Q8W & 48 & 0.65 (0.39 to 1.09$)$ & 0.105 \\
\hline
\end{tabular}


Table 1. Cont.

\begin{tabular}{|c|c|c|c|c|c|c|}
\hline Trial & \multicolumn{3}{|c|}{ Treatment Arm } & Subjects & $\begin{array}{c}\text { Relative Reduction } \\
\text { vs. Placebo }\end{array}$ & $p$ Value vs. \\
\hline \multirow{3}{*}{ Ohta et al., 2018} & \multirow{3}{*}{\multicolumn{2}{|c|}{$\begin{array}{l}\text { [CALIMA Japan] High-dosage ICS } \\
\text { plus LABA with baseline blood } \\
\text { eosinophils } \geq 300 \text { cells per uL }\end{array}$}} & Placebo & 16 & - & - \\
\hline & & & Benralizumab $30 \mathrm{mg}$ Q4W & 15 & 0.34 (0.11 to 0.99$)$ & \\
\hline & & & Benralizumab $30 \mathrm{mg}$ Q8W & 15 & $0.17(0.05$ to 0.60$)$ & \\
\hline \multirow{18}{*}{ Chipps et al., 2018} & \multirow{3}{*}{\multicolumn{2}{|c|}{$\begin{array}{l}\text { Met atopy and IgE } \\
30-700 \mathrm{kU} / \mathrm{L} \text { criteria }\end{array}$}} & Placebo & 179 & - & - \\
\hline & & & Benralizumab $30 \mathrm{mg}$ Q4W & 153 & $0.50(0.36$ to 0.69$)$ & $<0.0001$ \\
\hline & & & Benralizumab $30 \mathrm{mg}$ Q8W & 185 & 0.54 (0.39 to 0.74$)$ & 0.0002 \\
\hline & \multirow{3}{*}{\multicolumn{2}{|c|}{$\begin{array}{l}\text { Did not meet atopy and IgE } \\
\quad 30-700 \mathrm{kU} / \mathrm{L} \text { criteria }\end{array}$}} & Placebo & 336 & & \\
\hline & & & Benralizumab $30 \mathrm{mg}$ Q4W & 363 & 0.64 (0.51 to 0.81$)$ & 0.0002 \\
\hline & & & Benralizumab $30 \mathrm{mg}$ Q8W & 321 & $0.61(0.47$ to 0.78$)$ & $<0.0001$ \\
\hline & \multirow{3}{*}{\multicolumn{2}{|c|}{$\operatorname{IgE}$ high $(\geq 150 \mathrm{kU} / \mathrm{L})$}} & Placebo & 304 & & \\
\hline & & & Benralizumab $30 \mathrm{mg}$ Q4W & 304 & 0.64 (0.50 to 0.82$)$ & 0.0004 \\
\hline & & & Benralizumab $30 \mathrm{mg}$ Q8W & 297 & $0.58(0.45$ to 0.75$)$ & $<0.0001$ \\
\hline & \multirow{3}{*}{\multicolumn{2}{|c|}{$\operatorname{IgE}$ low $(<150 \mathrm{kU} / \mathrm{L})$}} & Placebo & 206 & & \\
\hline & & & Benralizumab $30 \mathrm{mg}$ Q4W & 207 & 0.54 (0.40 to 0.73$)$ & $<0.0001$ \\
\hline & & & Benralizumab $30 \mathrm{mg}$ Q8W & 199 & 0.57 (0.41 to 0.78$)$ & 0.0004 \\
\hline & \multirow{3}{*}{\multicolumn{2}{|c|}{ With atopy }} & Placebo & 316 & & \\
\hline & & & Benralizumab $30 \mathrm{mg}$ Q4W & 307 & 0.64 (0.50 to 0.82$)$ & 0.0004 \\
\hline & & & Benralizumab $30 \mathrm{mg}$ Q8W & 318 & $0.60(0.47$ to 0.77$)$ & $<0.0001$ \\
\hline & \multirow{3}{*}{\multicolumn{2}{|c|}{ Without atopy }} & Placebo & 193 & & \\
\hline & & & Benralizumab $30 \mathrm{mg}$ Q4W & 201 & $0.52(0.39$ to 0.71$)$ & $<0.0001$ \\
\hline & & & Benralizumab $30 \mathrm{mg}$ Q8W & 181 & 0.54 (0.39 to 0.74$)$ & 0.0002 \\
\hline \multirow{8}{*}{ Jackson et al., 2020} & \multirow{8}{*}{$\begin{array}{l}\text { Serum IgE } \\
\text { concentration } \\
(\mathrm{kU} / \mathrm{L})\end{array}$} & \multirow{2}{*}{$<62.0$} & Placebo & 75 & - & - \\
\hline & & & Benralizumab Q8W & 73 & $0.51(0.31-0.84)$ & 0.0079 \\
\hline & & \multirow{2}{*}{$\geq 62.0$ to $<176.2$} & Placebo & 112 & - & - \\
\hline & & & Benralizumab Q8W & 109 & $0.47(0.31-0.72)$ & 0.0004 \\
\hline & & \multirow{2}{*}{$\begin{array}{l}\geq 176.2 \\
\text { to }<453.4\end{array}$} & Placebo & 125 & - & - \\
\hline & & & Benralizumab Q8W & 106 & $0.52(0.35-0.76)$ & 0.0008 \\
\hline & & \multirow{2}{*}{$>453.4$} & Placebo & 129 & - & - \\
\hline & & & Benralizumab Q8W & 128 & $0.56(0.37-0.84)$ & 0.0057 \\
\hline \multirow{4}{*}{ Chipps et al., 2020} & \multirow{2}{*}{\multicolumn{2}{|c|}{$\mathrm{FAO}+{ }^{3}$}} & Placebo & 308 & - & - \\
\hline & & & Benralizumab Q8W & 313 & $0.56(0.44-0.71)$ & $<0.0001$ \\
\hline & \multirow{2}{*}{\multicolumn{2}{|c|}{$\mathrm{FAO}-$}} & Placebo & 193 & - & - \\
\hline & & & Benralizumab Q8W & 176 & $0.58(0.41-0.83)$ & 0.003 \\
\hline \multirow{12}{*}{ DuBuske et al., 2018} & \multirow{3}{*}{\multicolumn{2}{|c|}{ Winter }} & Benralizumab Q4W & 505 & $0.63(0.49-0.81)$ & $<0.001$ \\
\hline & & & Benralizumab Q8W & 495 & $0.60(0.46-0.78)$ & $<0.001$ \\
\hline & & & Placebo & 513 & - & - \\
\hline & \multirow{3}{*}{\multicolumn{2}{|c|}{ Spring }} & Benralizumab Q4W & 505 & $0.60(0.44-0.81)$ & $<0.001$ \\
\hline & & & Benralizumab Q8W & 490 & $0.50(0.36-0.69)$ & $<0.001$ \\
\hline & & & Placebo & 504 & - & - \\
\hline & \multirow{3}{*}{\multicolumn{2}{|c|}{ Summer }} & Benralizumab Q4W & 508 & $0.54(0.39-0.76)$ & $<0.001$ \\
\hline & & & Benralizumab Q8W & 487 & $0.55(0.39-0.78)$ & $<0.001$ \\
\hline & & & Placebo & 500 & - & - \\
\hline & & & Benralizumab Q4W & 506 & $0.57(0.44-0.74)$ & $<0.001$ \\
\hline & & & Benralizumab Q8W & 493 & $0.54(0.42-0.71)$ & $<0.001$ \\
\hline & & & Placebo & 510 & - & - \\
\hline
\end{tabular}

\footnotetext{
${ }^{1}$ Rate ratio vs. placebo; ${ }^{2} 80 \%$ Confidence Interval; ${ }^{3} \mathrm{FAO}+$ and FAO- are defined as $<70 \%$ or $\geq 70 \%$ of a ratio $\left({ }^{*} 100\right)$ of postbronchodilator $\mathrm{FEV}_{1}$ to FVC, respectively, at baseline, estimates calculated via a repeated measures model, with adjustment for study code, treatment, baseline value, region, OCS use at time of randomization, visit, and visit * treatment.
} 


\subsubsection{Tezepelumab}

The double-blind, randomized, 52-week, Phase Ilb PATHWAY study assessed the efficacy and safety of three dose levels of tezepelumab administered SC versus placebo in patients with uncontrolled asthma despite treatment with medium- to high-dose ICS and a LABA [12]. Treatment with tezepelumab resulted in significant reductions in the primary endpoint of annual asthma exacerbation rate (AAER) at Week 52 (62-71\%, depending on the dose). A post hoc analysis of the pooled tezepelumab cohort showed AAER reductions versus placebo ranging from $55 \%$ to $83 \%$. These reductions occurred irrespective of baseline levels of several type 2 inflammation biomarkers, including FeNO, blood eosinophils, IL 5, IL-13, and IgE [30]. NAVIGATOR is a Phase III, multicenter, randomized, doubleblind, parallel-group, placebo-controlled study designed to evaluate the efficacy and safety of regular SC administration of tezepelumab $210 \mathrm{mg}$ Q4W for 52 weeks in adult and adolescent patients with severe, uncontrolled asthma [20]. Tezepelumab reduced the AAER over 52 weeks versus placebo by $56 \%$ (RR, $0.44 ; 95 \%$ CI, $0.37-0.53$ ) in the overall study population and by $41 \%(\mathrm{RR}, 0.59 ; 95 \% \mathrm{CI}, 0.46-0.75)$ in patients with a baseline blood eosinophil count $<300$ cells $/ \mu \mathrm{L}$ ( $p<0.001$ for both). (Table 2 ).

\subsection{Forced Expiratory Volume in $1 \mathrm{~s}$}

\subsubsection{Benralizumab}

The Cochrane review which included Bleeker 2016, Castro 2014, and Fitzgerald 2016 demonstrated benralizumab increased forced expiratory volume in $1 \mathrm{~s}$ (FEV1) by $0.10 \mathrm{~L}$ ( $95 \%$ confidence interval 0.05 to 0.14 ) vs. placebo $\left(n=2355, \mathrm{I}^{2}=17 \%\right)$. Subgroup analysis indicated the differences between eosinophilic group ( $\mathrm{MD}=0.13 \mathrm{~L}, 95 \% \mathrm{CI} 0.08$ to 0.19 ) and non-eosinophilic group ( $0.03 \mathrm{~L}, 95 \% \mathrm{CI}-0.03$ to 0.10 .) However, when Goldman et al. applied an eosinophil cutoff of $\geq 150$ cells/ $\mu \mathrm{L}$ [24], the group of blood eosinophil counts $\geq 150$ demonstrated statistically significant in both CALIMA (MD $=0.116 \mathrm{~L}, 95 \% \mathrm{CI} 0.041$ to $0.191, p=0.0002$ ) [8] and SIROCCO [7] studies (MD $=0.163 \mathrm{~L}, 95 \% \mathrm{CI}, 0.087$ to 0.239 , $p<0.001$ ). (Other than eosinophilic subgroups, ethnicity, atopic status, IgE, and fixed airflow obstructions (FAO) were analyzed in following studies. Ohta et al. indicated benralizumab increased FEV1 by $0.334 \mathrm{~L}$ (Q4W; 95\% CI 0.020-0.647) and $0.198 \mathrm{~L}$ (q8w; 95\% CI -0.118 to 0.514 ) in Japanese patients [25]. Chipps demonstrated benralizumab increased FEV1 significantly regardless of serum IgE concentrations and atopic status [26]. Differences has shown between $\mathrm{FAO}+$ group and $\mathrm{FAO}-$ group in Chipps et al. study $(0.159 \mathrm{~L}, 95 \% \mathrm{CI} 0.082$ to $0.236, p<0.0001$ ) vs. $0.103 \mathrm{~L}, 95 \% \mathrm{CI},-0.008$ to $0.215, p=0.0218$ )] [28]. (Table 3).

Table 2. Summary of Annual Exacerbation rate-tezepelumab.

\begin{tabular}{|c|c|c|c|c|c|}
\hline Trial & \multicolumn{2}{|c|}{ Reatment Arm } & Subjects & $\begin{array}{l}\text { Relative Reduction } \\
\text { vs. Placebo }\end{array}$ & $\begin{array}{c}p \text { Value vs. } \\
\text { Placebo }\end{array}$ \\
\hline \multirow{12}{*}{ Corren et al., 2017} & \multirow{4}{*}{ Total } & Placebo & 138 & - & - \\
\hline & & Tezepelumab 70 mg q4w & 138 & $0.62(0.42-0.75)$ & $<0.001$ \\
\hline & & Tezepelumab 210 mg q4w & 137 & $0.71(0.54-0.82)$ & $<0.001$ \\
\hline & & Tezepelumab 280 mg q2w & 137 & $0.66(0.47-0.79)$ & $<0.001$ \\
\hline & \multirow{4}{*}{$\begin{array}{c}\geq 250 \text { Eosinophils per } \\
\text { uL }\end{array}$} & Placebo & 78 & - & - \\
\hline & & Tezepelumab 70 mg q4w & 80 & $0.65(0.30-0.82)$ & 0.003 \\
\hline & & Tezepelumab 210 mg q4w & 76 & $0.65(0.27-0.83)$ & 0.005 \\
\hline & & Tezepelumab 280 mg q2w & 76 & $0.72(0.40-0.87)$ & 0.001 \\
\hline & \multirow{4}{*}{$\begin{array}{c}<250 \text { Eosinophils per } \\
\text { uL }\end{array}$} & Placebo & 60 & - & - \\
\hline & & Tezepelumab 70 mg q4w & 58 & $0.60(0.12-0.81)$ & 0.022 \\
\hline & & Tezepelumab 210 mg q4w & 61 & $0.79(0.48-0.92)$ & $<0.001$ \\
\hline & & Tezepelumab 280 mg q2w & 61 & $0.58(0.11-0.80)$ & 0.024 \\
\hline
\end{tabular}


Table 2. Cont.

\begin{tabular}{|c|c|c|c|c|c|}
\hline Trial & \multicolumn{2}{|c|}{ Reatment Arm } & Subjects & $\begin{array}{l}\text { Relative Reduction } \\
\text { vs. Placebo }\end{array}$ & $\begin{array}{c}p \text { Value vs. } \\
\text { Placebo }\end{array}$ \\
\hline \multirow{4}{*}{ Emson et al., 2020} & \multirow{2}{*}{$\mathrm{NP}+$} & Placebo & 18 & - & \\
\hline & & Tezepelumab & 23 & $0.25(0.07-0.85)$ & \\
\hline & \multirow{2}{*}{ NP- } & Placebo & 117 & - & \\
\hline & & Tezepelumab & 112 & $0.27(0.14-0.53)$ & \\
\hline \multirow{12}{*}{$\begin{array}{l}\text { Menzies-Gow } \\
\text { et al., } 2021\end{array}$} & \multirow{2}{*}{$\begin{array}{c}\geq 300 \text { Eosinophils per } \\
\text { uL }\end{array}$} & Placebo & 222 & - & \\
\hline & & Tezepelumab & 219 & $0.30(0.22-0.40)$ & \\
\hline & \multirow{2}{*}{$\begin{array}{c}<300 \text { Eosinophils per } \\
\text { uL }\end{array}$} & Placebo & 309 & - & \\
\hline & & Tezepelumab & 309 & $0.59(0.46-0.75)$ & \\
\hline & \multirow{2}{*}{$\begin{array}{c}\geq 150 \text { Eosinophils per } \\
\text { uL }\end{array}$} & Placebo & 393 & - & \\
\hline & & Tezepelumab & 390 & $0.39(0.32-0.49)$ & \\
\hline & \multirow{2}{*}{$\begin{array}{c}<150 \text { Eosinophils per } \\
\text { uL }\end{array}$} & Placebo & 138 & - & \\
\hline & & Tezepelumab & 138 & $0.61(0.42-0.88)$ & \\
\hline & \multirow{2}{*}{$\mathrm{FeNO} \geq 25$} & Placebo & 307 & - & \\
\hline & & Tezepelumab & 309 & $0.32(0.25-0.42)$ & \\
\hline & \multirow{2}{*}{$\mathrm{FeNO}<25$} & Placebo & 220 & - & \\
\hline & & Tezepelumab & 213 & $0.68(0.51-0.92)$ & \\
\hline
\end{tabular}

Table 3. Summary of $\mathrm{FEV}_{1}$ — benralizumab.

\begin{tabular}{|c|c|c|c|c|c|c|c|}
\hline Trial & \multicolumn{3}{|c|}{ Treatment Arms } & $\mathbf{N}$ & $\begin{array}{l}\text { Difference vs. } \\
\text { Placebo }\end{array}$ & $\begin{array}{c}\text { Difference vs. } \\
\text { Placebo }(95 \% \text { CI })\end{array}$ & $p$-Value \\
\hline \multirow{3}{*}{ Bleecker et al., 2016} & \multirow{7}{*}{\multicolumn{2}{|c|}{ Eosinophilic }} & Placebo & 261 & - & - & - \\
\hline & & & Benralizumab 30 mg Q4W & 271 & 0.106 & 0.016 to 0.196 & 0.0215 \\
\hline & & & Benralizumab 30 mg Q8W & 264 & 0.159 & 0.068 to 0.249 & 0.0006 \\
\hline \multirow{3}{*}{ FitzGerald et al., 2016} & & & Placebo & 244 & - & - & - \\
\hline & & & Benralizumab 30 mg Q4W & 238 & 0.125 & 0.037 to 0.213 & 0.0054 \\
\hline & & & Benralizumab 30 mg Q8W & 238 & 0.116 & 0.028 to 0.204 & 0.0102 \\
\hline Castro et al., 2014 & & & Benralizumab $20 \mathrm{mg}$ & 48 & 0.23 & 0.11 to 0.36 & 0.019 \\
\hline Subtotal (95\% CI) & & & & & 0.13 & 0.08 to 0.19 & NR \\
\hline \multirow{3}{*}{ Bleecker et al., 2016} & \multirow{6}{*}{\multicolumn{2}{|c|}{ Non-eosinophilic }} & Placebo & 138 & - & - & - \\
\hline & & & Benralizumab 30 mg Q4W & 120 & -0.025 & -0.134 to 0.083 & 0.6438 \\
\hline & & & Benralizumab 30 mg Q8W & 129 & 0.102 & 0.003 to 0.208 & 0.568 \\
\hline \multirow{3}{*}{ FitzGerald et al., 2016} & & & Placebo & 116 & - & - & - \\
\hline & & & Benralizumab 30 mg Q4W & 114 & 0.064 & -0.049 to 0.176 & 0.2676 \\
\hline & & & Benralizumab 30 mg Q8W & 121 & -0.015 & -0.127 to 0.096 & 0.7863 \\
\hline Subtotal $(95 \% \mathrm{CI})$ & & & & & 0.03 & -0.03 to 0.10 & NR \\
\hline Total $(95 \%$ CI $)$ & & & & & 0.10 & 0.05 to 0.14 & NR \\
\hline \multirow{8}{*}{ Goldman et al., 2017} & \multirow{4}{*}{$\begin{array}{c}\text { blood } \\
\text { eosinophils } \geq \\
150 \text { cells per } \mathrm{uL}\end{array}$} & \multirow{2}{*}{ [SIROCCO] } & Placebo & 300 & - & - & - \\
\hline & & & Benralizumab 30 mg Q8W & 323 & 0.163 & 0.087 to 0.239 & $<0.001$ \\
\hline & & \multirow{2}{*}{ [CALIMA] } & Placebo & 308 & - & - & - \\
\hline & & & Benralizumab 30 mg Q8W & 298 & 0.116 & 0.041 to 0.191 & 0.002 \\
\hline & \multirow{4}{*}{$\begin{array}{c}\text { blood } \\
\text { eosinophils }<150 \\
\text { cells per } u L\end{array}$} & \multirow{2}{*}{ [SIROCCO] } & Placebo & 72 & - & - & - \\
\hline & & & Benralizumab 30 mg Q8W & 46 & 0.140 & -0.045 to 0.325 & 0.136 \\
\hline & & \multirow{2}{*}{ [CALIMA] } & Placebo & 37 & - & - & - \\
\hline & & & Benralizumab 30 mg Q8W & 46 & 0.131 & -0.306 to 0.045 & 0.142 \\
\hline
\end{tabular}


Table 3. Cont.

\begin{tabular}{|c|c|c|c|c|c|c|}
\hline Trial & \multicolumn{2}{|c|}{ Treatment Arms } & \multirow{2}{*}{$\begin{array}{l}\mathbf{N} \\
16\end{array}$} & \multirow{2}{*}{$\begin{array}{c}\text { Difference vs. } \\
\text { Placebo } \\
-\end{array}$} & \multirow{2}{*}{$\begin{array}{c}\text { Difference vs. } \\
\text { Placebo (95\% CI) } \\
-\end{array}$} & \multirow{2}{*}{$\frac{p \text {-Value }}{-}$} \\
\hline \multirow{3}{*}{ Ohta et al., 2018} & \multirow{3}{*}{$\begin{array}{l}\text { [CALIMA Japan] High-dosage ICS } \\
\text { plus LABA with baseline blood } \\
\text { eosinophils } \geq 300 \text { cells per uL }\end{array}$} & Placebo & & & & \\
\hline & & Benralizumab $30 \mathrm{mg}$ Q4W & 15 & 0.334 & 0.020 to 0.647 & \\
\hline & & Benralizumab $30 \mathrm{mg}$ Q8W & 15 & 0.198 & -0.118 to 0.514 & \\
\hline \multirow{18}{*}{ Chipps et al., 2018} & \multirow{3}{*}{$\begin{array}{l}\text { Met atopy and IgE } \\
\text { 30-700 kU/L criteria }\end{array}$} & Placebo & 178 & - & - & - \\
\hline & & Benralizumab $30 \mathrm{mg}$ Q4W & 149 & 0.129 & 0.017 to 0.241 & 0.0244 \\
\hline & & Benralizumab $30 \mathrm{mg}$ Q8W & 184 & 0.125 & 0.018 to 0.232 & 0.0218 \\
\hline & \multirow{3}{*}{$\begin{array}{l}\text { Did not meet atopy and IgE } \\
\quad 30-700 \mathrm{kU} / \mathrm{L} \text { criteria }\end{array}$} & Placebo & 327 & - & - & - \\
\hline & & Benralizumab $30 \mathrm{mg}$ Q4W & 360 & 0.114 & 0.040 to 0.187 & 0.0024 \\
\hline & & Benralizumab $30 \mathrm{mg}$ Q8W & 318 & 0.152 & 0.076 to 0.228 & $<0.0001$ \\
\hline & \multirow{3}{*}{$\operatorname{IgE}$ high $(\geq 150 \mathrm{kU} / \mathrm{L})$} & Placebo & 301 & - & - & - \\
\hline & & Benralizumab $30 \mathrm{mg}$ Q4W & 299 & 0.120 & 0.038 to 0.202 & 0.0042 \\
\hline & & Benralizumab $30 \mathrm{mg}$ Q8W & 296 & 0.123 & 0.041 to 0.205 & 0.0034 \\
\hline & \multirow{3}{*}{$\operatorname{IgE}$ low $(<150 \mathrm{kU} / \mathrm{L})$} & Placebo & 200 & - & - & - \\
\hline & & Benralizumab $30 \mathrm{mg}$ Q4W & 205 & 0.098 & 0.004 to 0.191 & 0.0405 \\
\hline & & Benralizumab $30 \mathrm{mg}$ Q8W & 197 & 0.138 & 0.044 to 0.233 & 0.0041 \\
\hline & \multirow{3}{*}{ With atopy } & Placebo & 314 & - & - & - \\
\hline & & Benralizumab $30 \mathrm{mg}$ Q4W & 303 & 0.103 & 0.022 to 0.184 & 0.0124 \\
\hline & & Benralizumab $30 \mathrm{mg}$ Q8W & 316 & 0.114 & 0.033 to 0.194 & 0.0056 \\
\hline & \multirow{3}{*}{ Without atopy } & Placebo & 186 & - & - & - \\
\hline & & Benralizumab $30 \mathrm{mg}$ Q4W & 198 & 0.148 & 0.053 to 0.242 & 0.0021 \\
\hline & & Benralizumab $30 \mathrm{mg}$ Q8W & 180 & 0.181 & 0.185 to 0.278 & 0.0002 \\
\hline \multirow{4}{*}{ Chipps et al., 2020} & \multirow{2}{*}{$\mathrm{FAO}+$} & Placebo & 304 & - & - & - \\
\hline & & Benralizumab q8w & 312 & 0.159 & 0.082 to 0.236 & $<0.0001$ \\
\hline & \multirow{2}{*}{ FAO- } & Placebo & 190 & - & - & - \\
\hline & & Benralizumab q8w & 175 & 0.103 & -0.008 to 0.215 & 0.0699 \\
\hline
\end{tabular}

\subsubsection{Tezepelumab}

Nominally significant improvements in prebronchodilator FEV1 versus placebo were observed in all tezepelumab groups from Week 4 through to the end of the study (120-150 mL at Week 52) [12]. At Week 52, differences in improvements from baseline in key secondary endpoints versus placebo in the overall population were: Prebronchodilator FEV1: $130 \mathrm{~mL}$ (95\% CI, 80-180 mL; $p<0.001$ ) [20], Improvements in prebronchodilator FEV1 versus placebo were observed at 2 weeks (first post baseline assessment) and were sustained throughout the treatment period. (Table 4).

\subsection{Asthma Control and Patient-Reported Outcomes Asthma Control Questionnaire}

3.4.1. Asthma Quality of Life Questionnaire

Benralizumab

Widely used patient-reported outcomes (PROs) in asthma treatments are the Asthma Control Questionnaire (ACQ) [31] and the Asthma Quality of Life Questionnaire (AQLQ) [32]. When using ACQ instrument, the Cochrane review which included Bleeker 2016, Castro 2014, and Fitzgerald 2016 demonstrated benralizumab increased HRQoL by -0.20 ( $95 \%$ CI -0.29 to -0.11 .) vs. placebo in both eosinophilic and non-eosinophilic participants [16]. Moreover, Goldman et al. demonstrated for patients with blood eosinophil counts $\geq 150$ cells $/ \mu \mathrm{L}$, decreases in ACQ-6 scores in comparison with placebo were observed in both the SIROCCO $(-0.15 ; 95 \% \mathrm{CI}-0.31$ to $0.02 ; p=0.084)$ and CALIMA studies $(-0.22$; $95 \% \mathrm{CI}-0.39$ to $-0.06 ; p=0.008$.) However, when using AQLQ instrument, the Cochrane review which included Bleeker 2016, Castro 2014, and Fitzgerald 2016 only demonstrated the increase in eosinophilic participants ( $\mathrm{MD}=0.23 ; 95 \% \mathrm{CI} 0.11$ to 0.35 ) vs. placebo [16]. For patients with blood eosinophil counts $<150$ cells $/ \mu \mathrm{L}$, improvements in AQLQ(S)+12 
and ACQ-6 scores were observed only in the SIROCCO study ( $p=0.056$ ) [24]. Chipps et al. evaluated fixed airflow obstruction (FAO) influence on benralizumab treatment response. The prevalence was 63\% (935/1493) for FAO+. ACQ-6 score was numerically greater for FAO+ vs. FAO- patients ( $\mathrm{MD}=-0.33$ vs. -0.18$)$, so as $A Q L Q(S)+12$ score $(\mathrm{MD}=0.33$ vs. 0.17) [28]. Chipps demonstrated benralizumab increased HRQoL regardless of serum IgE concentrations and atopic status [26]. (Table 5).

Table 4. Summary of FEV1-tezepelumab.

\begin{tabular}{|c|c|c|c|c|c|c|}
\hline Trial & & tment Arms & $\mathbf{N}$ & $\begin{array}{l}\text { Difference vs. } \\
\text { Placebo }\end{array}$ & $\begin{array}{c}\text { Difference vs. } \\
\text { Placebo ( } 95 \% \text { CI) }\end{array}$ & $p$-Value \\
\hline \multirow{12}{*}{ Corren et al., 2017} & \multirow{4}{*}{ Total } & Placebo & 131 & - & & \\
\hline & & Low-dose Tezepelumab & 130 & 0.12 & 0.02 to 0.22 & 0.015 \\
\hline & & Medium-dose Tezepelumab & 121 & 0.13 & 0.03 to 0.23 & 0.009 \\
\hline & & High-dose Tezepelumab & 116 & 0.15 & 0.05 to 0.25 & 0.002 \\
\hline & \multirow{4}{*}{$\begin{array}{l}\geq 250 \text { Eosinophils } \\
\text { per uL }\end{array}$} & Placebo & 76 & - & - & - \\
\hline & & Tezepelumab 70 mg q4w & 77 & 0.16 & 0.03 to 0.29 & 0.014 \\
\hline & & Tezepelumab 210 mg q4w & 66 & 0.17 & 0.04 to 0.3 & 0.013 \\
\hline & & Tezepelumab 280 mg q2w & 63 & 0.21 & 0.07 to 0.34 & 0.003 \\
\hline & \multirow{4}{*}{$\begin{array}{l}<250 \text { Eosinophils } \\
\text { per uL }\end{array}$} & Placebo & 55 & - & - & - \\
\hline & & Tezepelumab 70 mg q4w & 53 & 0.04 & -0.11 to 0.19 & 0.580 \\
\hline & & Tezepelumab 210 mg q4w & 55 & 0.08 & -0.07 to 0.23 & 0.289 \\
\hline & & Tezepelumab 280 mg q2w & 53 & 0.08 & -0.07 to 0.23 & 0.275 \\
\hline \multirow{2}{*}{\multicolumn{2}{|c|}{ Menzies-Gow et al., 2021}} & Placebo & 531 & - & - & - \\
\hline & & Tezepelumab & 528 & 0.13 & 0.08 to 0.18 & $p<0.001$ \\
\hline
\end{tabular}

Table 5. Summary of ACQ-6 score, and AQLQ(S)+12 score-benralizumab.

\begin{tabular}{|c|c|c|c|c|c|c|c|c|}
\hline \multirow[b]{2}{*}{ Trial } & & \multirow[b]{2}{*}{ Treatment Arm } & \multicolumn{3}{|c|}{ ACQ-6 Score } & \multicolumn{3}{|c|}{ AQLQ (S) +12 Score } \\
\hline & & & $\mathbf{N}$ & Difference vs. Placebo & $\begin{array}{c}p- \\
\text { Value }\end{array}$ & $\mathbf{N}$ & $\begin{array}{l}\text { Difference vs. } \\
\text { Placebo }\end{array}$ & $\begin{array}{c}p- \\
\text { Value }\end{array}$ \\
\hline \multirow{2}{*}{$\begin{array}{l}\text { Bleecker } \\
\text { et al., } 2016\end{array}$} & \multirow{6}{*}{ Eosinophilic } & Placebo & 267 & - & - & 254 & - & - \\
\hline & & $\begin{array}{l}\text { Benralizumab q4w } \\
\text { Benralizumab q8w }\end{array}$ & $\begin{array}{l}274 \\
263\end{array}$ & $\begin{array}{l}-0.15(-0.34 \text { to } 0.04) \\
-0.29(-0.48 \text { to }-0.10)\end{array}$ & $\begin{array}{l}0.1107 \\
0.0028\end{array}$ & $\begin{array}{l}261 \\
252\end{array}$ & $\begin{array}{c}0.18(-0.02 \text { to } 0.37) \\
0.3(0.10 \text { to } 0.50)\end{array}$ & $\begin{array}{l}0.0811 \\
0.0036\end{array}$ \\
\hline \multirow{3}{*}{$\begin{array}{l}\text { FitzGerald } \\
\text { et al., } 2016\end{array}$} & & Placebo & 247 & - & - & 240 & - & - \\
\hline & & Benralizumab Q4W & 241 & $-0.19(-0.38$ to -0.01$)$ & 0.0425 & 233 & $0.16(-0.04$ to 0.37$)$ & 0.1194 \\
\hline & & BenralizumabQ8W & 239 & $-0.25(-0.44$ to -0.07$)$ & 0.0082 & 230 & $0.24(0.04$ to 0.45$)$ & 0.1194 \\
\hline $\begin{array}{l}\text { Castro } \\
\text { et al., } 2014\end{array}$ & & Benralizumab 20 mg & 35 & $-0.44(-0.75$ to -0.12$)$ & 0.079 & 34 & $0.44(0.06$ to 0.81$)$ & 0.134 \\
\hline $\begin{array}{l}\text { Subtotal } \\
(95 \% \mathrm{CI})\end{array}$ & & & & $-0.23(-0.34$ to -0.12$)$ & NR & & $0.23(0.11$ to 0.35$)$ & NR \\
\hline \multirow{3}{*}{$\begin{array}{l}\text { Bleecker } \\
\text { et al., } 2016\end{array}$} & \multirow{6}{*}{ Non-eosinophilic } & Placebo & 138 & - & - & & & \\
\hline & & Benralizumab q4w & 124 & $0(-0.27$ to 0.27$)$ & 0.9903 & & & \\
\hline & & Benralizumab q8w & 130 & $-0.22(-0.48$ to -0.05$)$ & 0.1066 & & - & \\
\hline \multirow{3}{*}{$\begin{array}{l}\text { FitzGerald } \\
\text { et al., } 2016\end{array}$} & & Placebo & 122 & - & - & & & \\
\hline & & Benralizumab Q4W & 116 & $-0.24(-0.51$ to 0.03$)$ & 0.0776 & & & \\
\hline & & Benralizumab Q8W & 125 & $-0.10(-0.37$ to -0.16$)$ & 0.4488 & & & \\
\hline $\begin{array}{l}\text { Subtotal } \\
(95 \% \mathrm{CI})\end{array}$ & & & & $-014(-0.30$ to 0.02$)$ & NR & & & \\
\hline $\begin{array}{c}\text { Total } \\
(95 \% \text { CI })\end{array}$ & & & & $-0.20(-0.29$ to -0.11$)$ & NR & & & \\
\hline
\end{tabular}


Table 5. Cont.

\begin{tabular}{|c|c|c|c|c|c|c|c|c|c|}
\hline \multirow{8}{*}{$\begin{array}{l}\text { Goldman } \\
\text { et al., } 2017\end{array}$} & \multirow{4}{*}{$\begin{array}{l}\text { blood } \\
\text { eosinophils } \geq 150 \\
\text { cells per uL }\end{array}$} & \multirow{2}{*}{ [SIROCCO] } & Placebo & 305 & - & - & 294 & - & - \\
\hline & & & Benralizumab Q8W & 321 & $\begin{array}{c}-0.15 \\
(-0.31 \text { to } 0.02)\end{array}$ & 0.084 & 307 & $\begin{array}{c}0.19 \\
(0.01 \text { to } 0.37)\end{array}$ & 0.0036 \\
\hline & & \multirow{2}{*}{ [CALIMA] } & Placebo & 314 & - & - & 305 & - & - \\
\hline & & & Benralizumab Q8W & 300 & $\begin{array}{c}-0.22 \\
(-0.39 \text { to }-0.06)\end{array}$ & 0.0008 & 292 & $\begin{array}{c}0.2 \\
(0.02 \text { to } 0.38)\end{array}$ & 0.029 \\
\hline & \multirow{4}{*}{$\begin{array}{l}\text { blood } \\
\text { eosinophils }<150 \\
\text { cells per uL }\end{array}$} & \multirow{2}{*}{ [SIROCCO] } & Placebo & 73 & & & 70 & & \\
\hline & & & $\begin{array}{c}\text { Benralizumab } 30 \\
\text { mg Q8W }\end{array}$ & 47 & $\begin{array}{c}-0.7 \\
(-1.15 \text { to }-0.25)\end{array}$ & 0.0003 & 46 & $\begin{array}{c}0.46 \\
(-0.01 \text { to } 0.94)\end{array}$ & 0.056 \\
\hline & & \multirow{2}{*}{ [CALIMA] } & Placebo & 40 & - & - & 39 & - & - \\
\hline & & & $\begin{array}{c}\text { Benralizumab } 30 \\
\text { mg Q8W }\end{array}$ & 48 & $\begin{array}{c}-0.07 \\
(-0.56 \text { to } 0.43) \\
\end{array}$ & 0.783 & 46 & $\begin{array}{c}-0.01 \\
(-0.48 \text { to } 0.47)\end{array}$ & 0.972 \\
\hline \multirow{4}{*}{$\begin{array}{l}\text { Chipps } \\
\text { et al., } 2020\end{array}$} & \multirow{2}{*}{ FAO+ } & & Placebo & 308 & - & - & 299 & - & - \\
\hline & & Benr & calizumab q8w & 311 & $-0.33(-0.49$ to -0.17$)$ & $<0.0001$ & 300 & 0.33 (0.15 to 0.51$)$ & 0.0003 \\
\hline & \multirow{2}{*}{ FAO- } & & Placebo & 192 & - & - & 181 & - & - \\
\hline & & Benr & alizumab q8w & 175 & $-0.18(-0.40$ to 0.04$)$ & 0.1096 & 166 & $0.17(-0.08$ to 0.41$)$ & 0.1894 \\
\hline \multirow{6}{*}{$\begin{array}{l}\text { Chipps } \\
\text { et al., } 2018\end{array}$} & \multirow{3}{*}{$\begin{array}{l}\text { Met atopy and } \\
\text { IgE } 30-700 \mathrm{kU} / \mathrm{L} \\
\text { criteria }\end{array}$} & & Placebo & 179 & - & - & 176 & - & - \\
\hline & & Benra & alizumab Q4W & 152 & $-0.33(-0.55$ to -0.11$)$ & 0.0038 & 147 & $0.28(0.04$ to 0.52$)$ & 0.0207 \\
\hline & & Benra & alizumab Q8W & 185 & $-0.34(-0.55$ to -0.13$)$ & 0.0017 & 176 & $0.27(0.04$ to 0.50$)$ & 0.0193 \\
\hline & \multirow{3}{*}{$\begin{array}{l}\text { Did not meet } \\
\text { atopy and IgE } \\
30-700 \mathrm{kU} / \mathrm{L} \\
\text { criteria }\end{array}$} & & Placebo & 335 & - & - & 318 & - & - \\
\hline & & Benro & alizumab Q4W & 363 & $-0.12(-0.28$ to 0.03$)$ & 0.1111 & 347 & $0.11(-0.06$ to 0.27$)$ & 0.2057 \\
\hline & & Benra & alizumab Q8W & 317 & $-0.26(-0.41$ to -0.10$)$ & 0.0016 & 306 & $0.27(0.10$ to 0.44$)$ & 0.0022 \\
\hline
\end{tabular}

Tezepelumab

Patient-reported outcomes (PROs) were evaluated in Asthma Control Questionnaire (ACQ) [31] and the Asthma Quality of Life Questionnaire (AQLQ) [32] instruments in Menzies-Gow et al. study for tezepelumab. At week 52, improvements were greater with tezepelumab than with placebo with respect to the scores on the ACQ- 6 (MD $=-0.33 ; 95 \% \mathrm{CI}$, -0.46 to $-0.20 ; p<0.001$ ), AQLQ ( $\mathrm{MD}=0.34 ; 95 \% \mathrm{CI}, 0.20$ to $0.47 ; p<0.001$ ) [20]. (Table 6).

Table 6. Summary of ACQ-6 score, and AQLQ(S)+12 score-tezepelumab.

\begin{tabular}{|c|c|c|c|c|c|c|c|c|}
\hline \multirow[b]{2}{*}{ Trial } & & \multirow[b]{2}{*}{ Treatment Arm } & \multicolumn{3}{|c|}{ ACQ-6 Score } & \multicolumn{3}{|c|}{ AQLQ (S) +12 Score } \\
\hline & & & $\mathbf{N}$ & Difference vs. Placebo & $p$-Value & $\mathbf{N}$ & $\begin{array}{l}\text { Difference vs. } \\
\text { Placebo }\end{array}$ & $p$-Value \\
\hline \multirow{2}{*}{\multicolumn{2}{|c|}{ Menzies-Gow et al. }} & Placebo & 528 & - & - & 254 & - & - \\
\hline & & Tezepelumab & 531 & $-0.33(-0.46$ to 0.20$)$ & $<0.001$ & 261 & $0.34(0.20$ to 0.47$)$ & $<0.001$ \\
\hline \multirow{12}{*}{ Corren et al., 2017} & \multirow{4}{*}{ Total } & Placebo & 53 & - & & 47 & - & \\
\hline & & Low-dose Tezepelumab & 52 & $-0.26(-0.52$ to 0.01$)$ & 0.059 & 51 & $0.14(-0.13$ to 0.42$)$ & 0.309 \\
\hline & & $\begin{array}{l}\text { Medium-Dose } \\
\text { Tezepelumab }\end{array}$ & 44 & $-0.29(-0.56$ to -0.01$)$ & 0.039 & 41 & $0.2(-0.09$ to 0.48$)$ & 0.185 \\
\hline & & High-dose Tezepelumab & 49 & $-0.31(-0.58$ to -0.04$)$ & 0.024 & 48 & 0.34 (0.06 to 0.63$)$ & 0.017 \\
\hline & \multirow{4}{*}{$\begin{array}{l}\geq 250 \\
\text { Eosinophils } \\
\text { per } \mathrm{uL}\end{array}$} & Placebo & 68 & - & & 62 & - & \\
\hline & & Tezepelumab 70 mg q4w & 70 & $-0.19(-0.49$ to 0.11$)$ & 0.207 & 69 & $0.15(-0.19$ to 0.48$)$ & 0.383 \\
\hline & & Tezepelumab $210 \mathrm{mg} \mathrm{q} 4 \mathrm{w}$ & 60 & $-0.48(-0.79$ to -0.17$)$ & 0.002 & 54 & $0.41(0.06$ to 0.76$)$ & 0.022 \\
\hline & & Tezepelumab $280 \mathrm{mg}$ q2w & 55 & $-0.27(-0.58$ to -0.05$)$ & 0.094 & 54 & $0.27(-0.08$ to 0.61$)$ & 0.134 \\
\hline & \multirow{4}{*}{$\begin{array}{l}<250 \\
\text { Eosinophils } \\
\text { per uL }\end{array}$} & Placebo & 44 & - & & 43 & - & \\
\hline & & Tezepelumab 70 mg q4w & 46 & $-0.19(-0.53$ to 0.14$)$ & 0.261 & 41 & $0.09(-0.25$ to 0.43$)$ & 0.610 \\
\hline & & Tezepelumab 210 mg q4w & 50 & $-0.22(-0.56$ to -0.11$)$ & 0.186 & 43 & $0.24(-0.10$ to 0.58$)$ & 0.173 \\
\hline & & Tezepelumab 280 mg q2w & 47 & $-0.36(-0.70$ to -0.02$)$ & 0.036 & 45 & $0.49(0.15$ to 0.83$)$ & 0.004 \\
\hline
\end{tabular}




\subsubsection{Emergency Room Visits/Unscheduled Physician Visits}

Benralizumab

The Cochrane review which included Bleeker 2016, and Fitzgerald 2016 indicated in eosinophilic participants, benralizumab had fewer exacerbations requiring department treatment or admission by $0.68(95 \% \mathrm{CI} 0.47$ to 0.98$)$ [16]. The reduction rate was statistically significant in Bleeker et al. study [7], but not in FitzGerald et al. study [8]. Chipps et al. demonstrated annual AER reductions associated with emergency department visits or hospitalizations were greater for $\mathrm{FAO}+$ vs. $\mathrm{FAO}-$ patients (rate ratio $[95 \% \mathrm{CI}]=0.55$ [0.33-0.91] and 0.70 [0.33-1.48], respectively) [28]. (Table 7).

Table 7. Summary of ED visits/hospitalization—benralizumab.

\begin{tabular}{|c|c|c|c|c|c|c|}
\hline Trial & \multicolumn{3}{|c|}{ Treatment Arm } & Subjects & $\begin{array}{l}\text { Relative Reduction } \\
\text { vs. Placebo }\end{array}$ & $p$ Value vs. Placebo \\
\hline \multirow{3}{*}{ Bleecker et al., 2016} & \multirow{6}{*}{\multicolumn{2}{|c|}{ Eosinophilic }} & Placebo & 267 & - & - \\
\hline & & & Benralizumab 30 mg Q4W & 275 & $0.61(0.33$ to 1.13$)$ & $<0.0001$ \\
\hline & & & Benralizumab 30 mg Q8W & 267 & $0.37(0.17$ to 0.79$)$ & $<0.0001$ \\
\hline \multirow{3}{*}{ FitzGerald et al., 2016} & & & Placebo & 248 & - & - \\
\hline & & & Benralizumab 30 mg Q4W & 241 & 0.93 (0.41 to 2.09$)$ & 0.0018 \\
\hline & & & Benralizumab $30 \mathrm{mg}$ Q8W & 239 & $1.23(0.55$ to 2.74$)$ & 0.0188 \\
\hline Total $(95 \% \mathrm{CI})$ & & & & & $0.68(0.47-0.98)$ & NR \\
\hline \multirow{4}{*}{ Chipps et al., 2020} & \multirow{2}{*}{\multicolumn{2}{|c|}{$\mathrm{FAO}+$}} & Placebo & 308 & & \\
\hline & & & Benralizumab Q8W & 313 & $0.55(0.33-0.91)$ & 0.0195 \\
\hline & \multirow{2}{*}{\multicolumn{2}{|c|}{ FAO- }} & Placebo & 193 & & \\
\hline & & & Benralizumab Q8W & 176 & $0.70(0.33-1.48)$ & 0.3514 \\
\hline \multirow{8}{*}{ Goldman et al., 2017} & \multirow{4}{*}{$\begin{array}{l}\text { baseline blood } \\
\text { eosinophils } \geq 150 \\
\text { cells per uL }\end{array}$} & \multirow{2}{*}{ [SIROCCO] } & Placebo & 306 & & \\
\hline & & & Benralizumab $30 \mathrm{mg}$ Q8W & 325 & $0.54(0.32-0.90)$ & 0.018 \\
\hline & & \multirow{2}{*}{ [CALIMA] } & Placebo & 315 & & \\
\hline & & & Benralizumab 30 mg Q8W & 300 & NC & NC \\
\hline & \multirow{4}{*}{$\begin{array}{l}\text { baseline blood } \\
\text { eosinophils }<150 \\
\text { cells per uL }\end{array}$} & \multirow{2}{*}{ [SIROCCO] } & Placebo & 74 & & \\
\hline & & & Benralizumab $30 \mathrm{mg}$ Q8W & 48 & $1.92(0.72-5.14)$ & 0.192 \\
\hline & & \multirow{2}{*}{ [CALIMA] } & Placebo & 40 & & \\
\hline & & & Benralizumab $30 \mathrm{mg}$ Q8W & 48 & NC & NC \\
\hline
\end{tabular}

Tezepelumab

Both the Corren et al., and Menzies-Gow et al. studies showed the reduction rates in hospitalizations and emergency department visits $[13,20]$. Tezepelumab $70 \mathrm{mg}$ Q4W led to a relative rate reduction in asthma exacerbations that required hospitalizations of up to $73 \%$ and all-cause ED of up to 56\% compared with placebo [13]. Menzies-Gow et al. demonstrated fewer rate of exacerbations that were associated with hospitalization or an emergency in tezepelumab (rate ratio, $0.21 ; 95 \%$ CI, 0.12 to 0.37 ) vs. placebo over a period of 52 weeks [20]. Among patients admitted to the hospital or the ED, those treated with tezepelumab reported fewer mean days in the hospital and ED compared with those who received placebo (hospital: 10 days vs. 23 days; ED: 1.4 days vs. 3.6 days). (Table 8).

\subsection{Safety}

\subsubsection{Benralizumab}

The most common serious adverse events associated with benralizumab were worsening asthma (3-4\%), pneumonia ( $<1 \%$ to $1 \%)$, and pneumonia caused by bacterial infection $(0-1 \%)$. The percentages of patients who had any on-treatment adverse event, any serious adverse event, or any adverse event leading to treatment discontinuation during BORA were similar between patients originally assigned benralizumab and those originally assigned placebo and between benralizumab treatment regimens. The percentage of patients who had any adverse event was similar between SIROCCO or CALIMA (71-75\%; benral- 
izumab group only) and BORA (65-71\%), as was the percentage of patients who had an adverse event that led to treatment discontinuation (2\% in SIROCCO and CALIMA vs. $2-3 \%$ in BORA). Goldman et al. found the overall adverse events frequency was similar between treatment groups and eosinophil count cohorts [24]. Busse et al. assessed the long-term safety and efficacy of benralizumab between 19 November 2014 and 6 July 2016, [9] The most common adverse events in all groups were viral upper respiratory tract infection (14-16\%) and worsening asthma (7-10\%) [9].

Table 8. Summary of ED visits/hospitalization-tezepelumab.

\begin{tabular}{ccccc}
\hline Trial & Treatment Arm & Subjects & Rate Ratio vs. Placebo & $p$ Value vs. Placebo \\
\cline { 2 - 5 } Corren et al., 2020 & $\begin{array}{c}\text { Placebo } \\
\text { Low-dose } \\
\text { tezepelumab }\end{array}$ & 138 & $0.44(0.14-1.41)$ & - \\
\cline { 2 - 5 } & $\begin{array}{c}\text { Medium-Dose } \\
\text { tezepelumab }\end{array}$ & 137 & $0.16(0.04-0.69)$ & - \\
\cline { 2 - 4 } & $\begin{array}{c}\text { High-dose } \\
\text { tezepelumab }\end{array}$ & 137 & $0.63(0.22-1.81)$ & - \\
\cline { 2 - 4 } & $\begin{array}{c}\text { Overall } \\
\text { tezepelumab }\end{array}$ & 412 & $0.40(0.17-0.97)$ & \\
\hline
\end{tabular}

\subsubsection{Tezepelumab}

Safety findings were similar between tezepelumab and placebo groups in both Corren et al. and Menzies-Gow et al. studies. Three serious adverse events occurred in Corren et al's study [12], two (pneumonia and stroke) occurred in the same patient using tezepelumab $70 \mathrm{mg} \mathrm{Q} 4 \mathrm{~W}$, and one (the Guillain-Barre syndrome) using tezepelumab $210 \mathrm{mg}$ Q4W. The discontinuation rates due to adverse events were $1.2 \%$ among patients receiving tezepelumab (five patients, including two in tezepelumab $210 \mathrm{mg}$ Q4W, and three in tezepelumab $280 \mathrm{mg} \mathrm{Q} 2 \mathrm{~W}$ ) and $0.7 \%$ in the placebo group (one patient). In Menzies-Gow et al's. study, the discontinuation rates was $2.1 \%$ in the tezepelumab group and $3.6 \%$ in the placebo group [20]. The most common adverse events were nasopharyngitis, upper respiratory tract infection, headache, and asthma (which was more frequently observed in the placebo group than in the tezepelumab group).

\section{Conclusions}

Benralizumab significantly reduced exacerbations, improved lung function, and increased patient report outcomes versus placebo. These clinical benefits were sustained long term ( 2 years). The annual exacerbation profile with benralizumab was similar to that with placebo in the 1-year pivotal studies. Long-term depletion of eosinophils with benralizumab was not associated with new safety risks after 2 years of exposure. Tezepelumab reduced annual exacerbations regardless of baseline levels of several type 2 inflammation biomarkers, including FeNO, blood eosinophils, IL 5, IL-13, and IgE. Lung function and health-related quality of life are both improved in tezepelumab among severe uncontrolled asthma patients, including those with low blood eosinophil counts.

Supplementary Materials: The following are available online at https: / www.mdpi.com/article/ 10.3390/life11080744/s1, Table S1. Search strategy in PICOS; Table S2. Search strategy in PubMed (search date: 19 April 2021); Table S3. Overview of Studies; Table S4. Overview of post analysis; Table S5. Baseline Characteristics; Table S6 Summary of Annual Exacerbation rate; Table S7. Summary of FEV1; Table S8. Summary of Asthma symptoms score; Table S9. Summary of ACQ-6 score, and AQLQ(S)+12 score; Table S10. Summary of ED visits/hospitalization; Table S11. Summary of Observational Studies.

Funding: This research received no external funding.

Institutional Review Board Statement: Not applicable. 
Informed Consent Statement: Not applicable.

Data Availability Statement: Data is contained within the article or supplementary material.

Acknowledgments: This study was supported by grants from the Far Eastern Memorial Hospital (numbers: FEMH-2019-C-028 and FEMH-2020-C-026).

Conflicts of Interest: The authors declare no conflict of interest.

\begin{tabular}{|c|c|}
\hline Abbreviations & \\
\hline Abbreviation & Definition \\
\hline A & Asian \\
\hline ACQ-6 & Asthma Control Questionnaire 6 \\
\hline $\mathrm{AE}$ & Adverse event \\
\hline AQLQ [S]+12 & $\begin{array}{l}\text { Asthma Quality of Life Questionnaire (standardized) for persons } \\
12 \text { years of age or older }\end{array}$ \\
\hline $\mathrm{B}$ & Black \\
\hline $\mathrm{BEC}$ & Blood eosinophil count \\
\hline $\mathrm{CI}$ & Confidence interval \\
\hline ED & Emergency department \\
\hline ELEN & Eosinophil/lymphocyte and eosinophil/neutrophil \\
\hline FAO & Fixed airflow obstruction \\
\hline $\mathrm{FEV}_{1}$ & Forced expiratory volume in $1 \mathrm{~s}$ \\
\hline FVC & Forced vital capacity \\
\hline $\mathrm{H}$ & Hispanic or Latino \\
\hline ICS & Inhaled corticosteroids \\
\hline $\operatorname{IgE}$ & Immunoglobulin \\
\hline ITT & Intent-to-treat \\
\hline $\mathrm{J}$ & Japan \\
\hline LABA & Long-acting $\beta_{2}$ agonists \\
\hline LS & Lease square \\
\hline M & Missing \\
\hline $\mathrm{N}$ & Native Hawaiian or other Pacific Islander \\
\hline NA & Not available \\
\hline $\mathrm{NC}$ & Not calculable \\
\hline NR & Not reported due to small sample size \\
\hline $\mathrm{O}$ & Other \\
\hline Q4W & Every 4 weeks \\
\hline Q8W & Every 8 weeks \\
\hline $\mathrm{S}$ & South Korea \\
\hline SAE & Serious adverse event \\
\hline W & White \\
\hline
\end{tabular}

\section{References}

1. Chung, K.F.; Wenzel, S.E.; Brozek, J.L.; Bush, A.; Castro, M.; Sterk, P.J.; Adcock, I.; Bateman, E.D.; Bel, E.H.; Bleecker, E.R.; et al. International ERS/ATS guidelines on definition, evaluation and treatment of severe asthma. Eur. Respir. J. 2013, 43, 343-373. [CrossRef]

2. Fahy, J.V. Type 2 inflammation in asthma-Present in most, absent in many. Nat. Rev. Immunol. 2015, 15, 57-65. [CrossRef] [PubMed]

3. Tan, R.; Liew, M.F.; Lim, H.F.; Leung, B.; Wong, W.F. Promises and challenges of biologics for severe asthma. Biochem. Pharmacol. 2020, 179, 114012. [CrossRef]

4. Dorey-Stein, Z.L.; Shenoy, K.V. Tezepelumab as an Emerging Therapeutic Option for the Treatment of Severe Asthma: Evidence to Date. Drug Des. Dev. Ther. 2021, 15, 331-338. [CrossRef]

5. Ayres, J.G.; Higgins, B.; Chilvers, E.; Ayre, G.; Blogg, M.; Fox, H. Efficacy and tolerability of anti-immunoglobulin E therapy with omalizumab in patients with poorly controlled (moderate-to-severe) allergic asthma. Allergy 2004, 59, 701-708. [CrossRef]

6. Pelaia, C.; Calabrese, C.; Vatrella, A.; Busceti, M.T.; Garofalo, E.; Lombardo, N.; Terracciano, R.; Pelaia, G. Benralizumab: From the Basic Mechanism of Action to the Potential Use in the Biological Therapy of Severe Eosinophilic Asthma. BioMed Res. Int. 2018, 2018, 1-9. [CrossRef] [PubMed] 
7. Bleecker, E.R.; FitzGerald, J.M.; Chanez, P.; Papi, A.; Weinstein, S.F.; Barker, P.; Sproule, S.; Gilmartin, G.; Aurivillius, M.; Werkström, V.; et al. Efficacy and safety of benralizumab for patients with severe asthma uncontrolled with high-dosage inhaled corticosteroids and long-acting beta2-agonists (SIROCCO): A randomised, multicentre, place-bo-controlled phase 3 trial. Lancet 2016, 388, 2115-2127. [CrossRef]

8. FitzGerald, J.M.; Bleecker, E.R.; Nair, P.; Korn, S.; Ohta, K.; Lommatzsch, M.; Ferguson, G.T.; Busse, W.W.; Barker, P.; Sproule, S.; et al. Benralizumab, an anti-interleukin-5 receptor $\alpha$ monoclonal antibody, as add-on treatment for patients with severe, uncontrolled, eosinophilic asthma (CALIMA): A randomised, double-blind, placebo-controlled phase 3 trial. Lancet 2016, 388, 2128-2141. [CrossRef]

9. Busse, W.W.; Bleecker, E.R.; FitzGerald, J.M.; Ferguson, G.T.; Barker, P.; Sproule, S.; Olsson, R.; Martin, U.J.; Goldman, M.; Yañez, A.; et al. Long-term safety and efficacy of benralizumab in patients with severe, uncontrolled asthma: 1-year results from the BORA phase 3 extension trial. Lancet Respir. Med. 2019, 7, 46-59. [CrossRef]

10. Nair, P.; Wenzel, S.; Rabe, K.F.; Bourdin, A.; Lugogo, N.L.; Kuna, P.; Barker, P.; Sproule, S.; Ponnarambil, S.; Goldman, M. Oral Glucocorticoid-Sparing Effect of Benralizumab in Severe Asthma. N. Engl. J. Med. 2017, 376, 2448-2458. [CrossRef]

11. Harrison, T.W.; Chanez, P.; Menzella, F.; Canonica, G.W.; Louis, R.; Cosio, B.G. Onset of effect and impact on health-related quality of life, exacerbation rate, lung function, and nasal polyposis symptoms for patients with severe eosinophilic asthma treated with benralizumab (ANDHI): A random-ised, controlled, phase 3b trial. Lancet Respir. Med. 2021, 9, 260-274. [CrossRef]

12. Corren, J.; Parnes, J.R.; Wang, L.; Mo, M.; Roseti, S.L.; Griffiths, J.M.; Van Der Merwe, R. Tezepelumab in Adults with Uncontrolled Asthma. N. Engl. J. Med. 2017, 377, 936-946. [CrossRef] [PubMed]

13. Harrison, T.W.; Chanez, P.; Menzella, F.; Canonica, G.W.; Louis, R.; Cosio, B.G. The effect of tezepelumab on hospitalizations and emergency department visits in patients with severe asthma. Ann. Allergy Asthma Immunol. 2020, 125, $211-214$.

14. Corren, J.; Gil, E.G.; Griffiths, J.M.; Parnes, J.R.; van der Merwe, R.; Sałapa, K.; O'Quinn, S. Tezepelumab improves patientreported outcomes in patients with severe, uncontrolled asthma in PATHWAY. Ann. Allergy Asthma Immunol. 2021, 126, 187-193. [CrossRef] [PubMed]

15. Hekking, P.-P.W.; Wener, R.R.; Amelink, M.; Zwinderman, A.H.; Bouvy, M.; Bel, E.H. The prevalence of severe refractory asthma. J. Allergy Clin. Immunol. 2015, 135, 896-902. [CrossRef]

16. Farne, H.A.; Wilson, A.; Powell, C.; Bax, L.; Milan, S.J. Anti-IL5 therapies for asthma. Cochrane Database Syst. Rev. 2017, 9, CD010834. [CrossRef]

17. Bourdin, A.; Husereau, D.; Molinari, N.; Golam, S.; Siddiqui, M.K.; Lindner, L.; Xu, X. Matching-adjusted indirect comparison of benralizumab versus interleukin-5 inhibitors for the treatment of severe asthma: A systematic review. Eur. Respir. J. 2018, 52, 1801393. [CrossRef] [PubMed]

18. Bourdin, A.; Husereau, D.; Molinari, N.; Golam, S.; Siddiqui, M.K.; Lindner, L.; Xu, X. Matching-adjusted comparison of oral corticosteroid reduction in asthma: Systematic review of biologics. Clin. Exp. Allergy 2020, 50, 442-452. [CrossRef]

19. Rogliani, P.; Calzetta, L.; Matera, M.G.; Laitano, R.; Ritondo, B.L.; Hanania, N.A.; Cazzola, M. Severe Asthma and Biological Therapy: When, Which, and for Whom. Pulm. Ther. 2020, 6, 47-66. [CrossRef] [PubMed]

20. Menzies-Gow, A.; Corren, J.; Bourdin, A.; Chupp, G.; Israel, E.; Wechsler, M.E.; Brightling, C.E.; Griffiths, J.M.; Hellqvist, Å.; Bowen, K.; et al. Tezepelumab in Adults and Adolescents with Severe, Uncontrolled Asthma. N. Engl. J. Med. 2021, 384, 1800-1809. [CrossRef]

21. Menzella, F.; Galeone, C.; Ruggiero, P.; Bagnasco, D.; Catellani, C.; Facciolongo, N. Biologics and Bronchial Thermoplasty for severe refractory asthma treatment: From eligibility criteria to real practice. A cross-sectional study. Pulm. Pharmacol. Ther. 2020, 60, 101874. [CrossRef] [PubMed]

22. Cook, A.; Harrington, J.; Simpson, J.L.; Wark, P. Mepolizumab asthma treatment failure due to refractory airway eosinophilia, which responded to benralizumab. Respirol. Case Rep. 2021, 9, e00743.

23. Padilla-Galo, A.; Levy-Abitbol, R.; Olveira, C.; Azcona, B.V.; Morales, M.P.; Rivas-Ruiz, F.; Tortajada-Goitia, B.; Moya-Carmona, I.; Levy-Naon, A. Real-life experience with benralizumab during 6 months. BMC Pulm. Med. 2020, 20, 184. [CrossRef] [PubMed]

24. Goldman, M.; Hirsch, I.; Zangrilli, J.G.; Newbold, P.; Xu, X. The association between blood eosinophil count and benralizumab efficacy for patients with se-vere, uncontrolled asthma: Subanalyses of the Phase III SIROCCO and CALIMA studies. Curr. Med. Res. Opin. 2017, 33, 1605-1613. [CrossRef]

25. Ohta, K.; Adachi, M.; Tohda, Y.; Kamei, T.; Kato, M.; Fitzgerald, J.M.; Takanuma, M.; Kakuno, T.; Imai, N.; Wu, Y.; et al. Efficacy and safety of benralizumab in Japanese patients with severe, uncontrolled eosinophilic asthma. Allergol. Int. 2018, 67, 266-272 [CrossRef]

26. Chipps, B.E.; Newbold, P.; Hirsch, I.; Trudo, F.; Goldman, M. Benralizumab efficacy by atopy status and serum immunoglobulin E for patients with severe, uncontrolled asthma. Ann. Allergy Asthma Immunol. 2018, 120, 504-511.e4. [CrossRef]

27. Jackson, D.J.; Humbert, M.; Hirsch, I.; Newbold, P.; Gil, E.G. Ability of Serum IgE Concentration to Predict Exacerbation Risk and Benralizumab Efficacy for Patients with Severe Eosinophilic Asthma. Adv. Ther. 2020, 37, 718-729. [CrossRef]

28. Chipps, B.E.; Hirsch, I.; Trudo, F.; Alacqua, M.; Zangrilli, J.G. Benralizumab efficacy for patients with fixed airflow obstruction and severe, uncontrolled eosinophilic asthma. Ann. Allergy Asthma Immunol. 2020, 124, 79-86. [CrossRef] [PubMed]

29. DuBuske, L.; Newbold, P.; Wu, Y.; Trudo, F. Seasonal variability of exacerbations of severe, uncontrolled eosinophilic asthma and clinical benefits of benralizumab. Allergy Asthma Proc. 2018, 39, 345-349. [CrossRef] 
30. Emson, C.; Corren, J.; Sałapa, K.; Hellqvist, Å.; Parnes, J.R.; Colice, G. Efficacy of Tezepelumab in Patients with Severe, Uncontrolled Asthma with and without Nasal Polyposis: A Post Hoc Analysis of the Phase 2b PATHWAY Study. J. Asthma Allergy 2021, 14, 91-99. [CrossRef]

31. Juniper, E.; O'byrne, P.; Guyatt, G.; Ferrie, P.; King, D. Development and validation of a questionnaire to measure asthma control. Eur. Respir. J. 1999, 14, 902-907. [CrossRef] [PubMed]

32. Juniper, E.F.; Guyatt, G.H.; Epstein, R.S.; Ferrie, P.J.; Jaeschke, R.; Hiller, T.K. Evaluation of impairment of health related quality of life in asthma: Development of a question-naire for use in clinical trials. Thorax 1992, 47, 76-83. [CrossRef] [PubMed] 